\title{
Stochastic Texture Image Estimators for Local Spatial Anisotropy and Its Variability
}

\author{
J. Scharcanski and C. T. J. Dodson
}

\begin{abstract}
A new image analysis technique is proposed for the evaluation of local anisotropy and its variability in stochastic texture images. It utilizes the gradient function to provide information on local anisotropy, from two-dimensional (2-D) density images for foil materials like polymer sheets, nonwoven textiles, and paper. Such images can be captured by radiography or light-transmission; results are reported for a range of paper structures, and show that the proposed technique is more robust to unfavorable imaging conditions than other approaches. The method has potential for on-line application to monitoring and control of anisotropy and its variability, as well as local density itself, in continuous manufacturing processes.
\end{abstract}

Index Terms-Anisotropy, density variability, machine control, monitoring, stochastic structures, texture image analysis.

\section{INTRODUCTION}

C EVERAL approaches have been considered to analyze stochastic texture images with respect to their anisotropy. Gradient based methods have been proposed [1], in particular, for texture analysis applications. However, such methods have limitations [9] because they rely only on local angular information, which may lead to imprecise anisotropy estimation; and also they are susceptible to mutual vector cancellation, which may affect the results of local gradient vector operations. However, these deficiencies found in the proposed gradient based methods can be overcome by considering anisotropy as a global feature, described in terms of the distribution of local gradient directions [12]. A limitation of this approach is the lack of information about local anisotropy variability. Most recent research on this topic is reported by Praast and Göttsching concerning the local orientation in fiber assemblies.

The modeling of the local anisotropic process, and how it affects the stochastic textures originating from it, has been intensively studied. The geometric optics of anisotropic fiber assemblies, originating stochastic textures, were studied by Charrier and Marchessault [4]. The early work of Corte and Kallmes [2], [3], laid the basis for statistical geometric representation of anisotropy in web-based materials like paper, and Perkins and Mark [6] emphasized the need for more than one parameter in characterizing anisotropy. Niskanen and Sadowski [5] compared different methods of measuring orientation in images of fiber assemblies, and Schaffnit et al. [10], [11], developed a

Manuscript received March 25, 1999; revised May 31, 2000. This work was supported in part by the CNPq (Brazilian National Research Council).

J. Scharcanski is with the Institute of Informatics, Federal University of Rio Grande do Sul, Porto Alegre, Brazil 91501-970.

C. T. J. Dodson is with the Department of Mathematics, UMIST, Manchester M60 1QD, U.K.

Publisher Item Identifier S 0018-9456(00)07031-5. model to analyze the effect of anisotropy on the mass variability in planar stochastic materials. These methods have been useful to understand better the underlying anisotropic process, and its effect on the stochastic textures. However, their application to direct anisotropy measurement is not trivial.

A different approach based on texture spectral density analysis in the Fourier domain was discussed in [9]. This method is discussed later, as well as some of its limitations for anisotropy detection in stochastic textures, and it is shown that these difficulties can be reduced by using our approach.

The next sections describe the method we propose for detection of anisotropy and its variability in stochastic textures. Finally, some experimental results, on texture images of paper samples are presented.

\section{LOCAL SPATIAL ANISOTROPY FROM IMAGE GRADIENTS}

We apply our methods to the anisotropy of a paper sample, estimated via analysis of its grey-level image, which may be obtained through different transmission imaging techniques. Initially, the image is convolved with a gradient operator, and at the gradient maxima we estimate the gradient magnitudes and orientations. This information is then used to estimate the anisotropy of the sample, and its spatial variability. The next sections describe the gradient operator we use, and our technique for anisotropy estimation. The anisotropy spatial variability is also estimated and constitutes a relevant feature for image discrimination.

\section{A. Detection of Local Image Gradients}

The isotropic operator for grey-level images is utilized to obtain the image gradients, represented by a local gradient vector $\mathrm{at}$ each image position $(x, y)$ [8]. The $3 \times 3$ version of this operator is described using the following pixel numbering convention:

$$
\left(\begin{array}{ccc}
A_{0} & A_{1} & A_{2} \\
A_{7} & g(x, y) & A_{3} \\
A_{6} & A_{5} & A_{4}
\end{array}\right) .
$$

Therefore, the magnitude of the gradient maximum at the image location $(x, y)$ is obtained by

$$
B(x, y)=\sqrt{\Delta H(x, y)^{2}+\Delta V(x, y)^{2}}
$$

where

$$
\begin{aligned}
& \Delta H(x, y) \\
& \quad=\frac{1}{K+2}\left[\left(A_{2}+K A_{3}+A_{4}\right)-\left(A_{0}+K A_{7}+A_{6}\right)\right]
\end{aligned}
$$




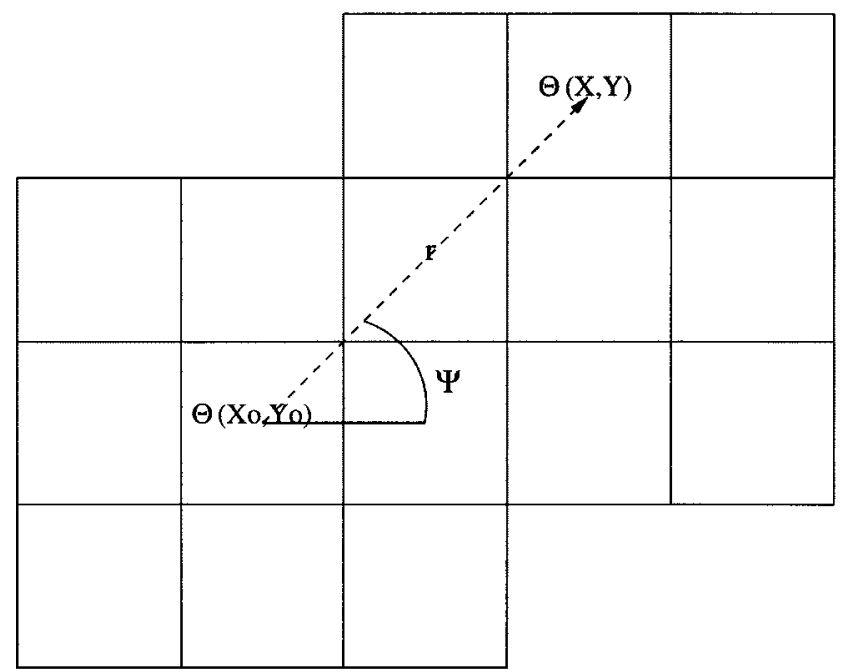

Fig. 1. Local angular difference for a given $r$ and $\psi$.

$$
\begin{aligned}
& \Delta V(x, y) \\
& \quad=\frac{1}{K+2}\left[\left(A_{0}+K A_{1}+A_{2}\right)-\left(A_{6}+K A_{5}+A_{4}\right)\right]
\end{aligned}
$$

and the direction $\theta$ of the gradient maximum at $(x, y)$ is given by

$$
\theta(x, y)=\tan ^{-1}\left(\frac{\Delta V(x, y)}{\Delta H(x, y)}\right)+\kappa \pi
$$

where $\kappa$ is an integer, and $K=\sqrt{2}$. This operator is isotropic because its sensitivity to horizontal, vertical, and diagonal step edges is the same. Therefore, at each image location $(x, y)$ there is the maximum gradient magnitude $B(x, y)$, and its orientation $\theta(x, y)$, i.e., forming a local gradient vector.

\section{B. Local Anisotropy and its Spatial Variability}

Let us consider two pixels $\left(x_{i}, y_{i}\right)$ and $\left(x_{j}, y_{j}\right)$, with their gradient magnitudes normalized to unity. These pixels are located at a distance $r$ from each other, with the line connecting them rotated of an angle $\Psi$ with respect to the horizontal axis (see Fig. 1). The inner product of the two gradient vectors associated with the pixels $\left(x_{i}, y_{i}\right)$ and $\left(x_{j}, y_{j}\right)$ is given by $\phi(r, \Psi)=\cos \left[\theta\left(x_{i}, y_{i}\right)-\theta\left(x_{j}, y_{j}\right)\right]$. Papermakers report the existence of an underlying weak anisotropic process favoring the direction of paper forming; $\phi(r, \Psi)$ tends to be higher when measured along the directions of anisotropy than along other directions, where gradients are less likely to be aligned. Based on this observation, we can estimate the local anisotropy and its variability, within different region sizes.

Local anisotropy is detected via the distribution of the average inner product values $\overline{\phi(r, \Psi)}$, obtained by varying $r$ and $\Psi$. The range of $r$ and $\Psi$ values defines a particular region size, i.e., the neighborhood where local anisotropy is analyzed

$$
\begin{aligned}
\overline{\phi(r, \Psi)}= & \frac{1}{M N} \sum_{y=1}^{y=M} \sum_{x=1}^{x=N} \cos [\theta(x, y) \\
& -\theta(x+r \cos \Psi, y+r \sin \Psi)]
\end{aligned}
$$

where $M \times N$ is the number of pixels within the image area under consideration.

The shape of these $\overline{\phi(r, \Psi)}$ distributions encode important structural information. The stronger the local anisotropy, the higher is the local alignment of gradient vectors, and more elliptical is the distribution of $\overline{\phi(r, \Psi)}$ values. A quantitative measure of how elliptical is the distribution may be provided by the eccentricity factor, denoted by $e$, which is detailed next [12].

1) Eccentricity of the Angular Distribution: The distribution of $\overline{\phi(r, \Psi)}$ values over all locations $(x, y)$, varying the distance $r$ and the angle $\Psi$, represents structural anisotropy. In the case where the values are equally probable in all angles $\Psi$, the sample is isotropic (i.e., cylindrically symmetric $\overline{\phi(r, \Psi)}$ distribution).

The distribution of $\phi(r, \Psi)$ in an anisotropic sample is asymmetric; it is approximately elliptical and determines two orthogonal axes of extremal variance. These axes coincide with eigenvectors of the $\overline{\phi(r, \Psi)}$ distribution of values, belonging to the two distinct eigenvalues $\left(\lambda_{\max }, \lambda_{\min }\right)$ of the covariance matrix. The eigenvalues define an ellipse with semimajor axes given by $\left(\lambda_{\max }, \lambda_{\min }\right)$, which is described by

$$
\frac{\left({\overline{\phi(r, \Psi)}}_{\lambda_{\max }}\right)^{2}}{\lambda_{\max }^{2}}+\frac{\left(\overline{\phi(r, \Psi)}_{\lambda_{\min }}\right)^{2}}{\lambda_{\min }^{2}}=1 .
$$

The eigenvalues are related to the eccentricity $e$ of this ellipse (and of the distribution). We represent the eccentricity simply as the ratio

$$
e^{2}=\frac{\lambda_{\max }^{2}}{\lambda_{\min }^{2}}
$$

where $e \geq 1$, and we need to determine $\lambda_{\min }$ and $\lambda_{\max }$.

In general, the $k$ th principal component direction of a distribution is along an eigenvector direction belonging to the $k$ th largest eigenvalue of the covariance matrix. Therefore, the eigenvalues can be calculated from the covariance matrix, which is defined in terms of the $\overline{\phi(r, \Psi)}$ projections along the horizontal and vertical directions (i.e., $\phi(r, \Psi)_{x}=\overline{\phi(r, \Psi)} \cos \Psi$ \left. and ${\overline{\phi(r, \Psi)_{y}}}_{y}=\overline{\phi(r, \Psi)} \sin \Psi\right)$, as well as their means $\mu_{x}^{r, \Psi}$ and $\mu_{y}^{r, \Psi}$

$$
C v=E\left\{\left(\overline{\phi(r, \Psi)}_{x}-\mu_{x}^{r, \Psi}\right)\left(\overline{\phi(r, \Psi)}_{y}-\mu_{y}^{r, \Psi}\right)\right\}
$$

or

$$
C v=\left[\begin{array}{ll}
v_{x x} & v_{x y} \\
v_{y x} & v_{y y}
\end{array}\right]
$$

where $v_{x y}$ is the covariance about the means $\mu_{x}^{r, \Psi}$ and $\mu_{y}^{r, \Psi}$.

This matrix is symmetric, i.e., $C v_{x y}=C v_{y x}$, which implies that its eigenvalues are all real and its eigenvectors are orthogonal. Also, because the bilinear form, $C v$ is positive semi-definite, its eigenvalues are positive or zero, and given by

$$
\operatorname{det}[C v-\lambda I]=0
$$

where $\lambda$ is the diagonal matrix of eigenvalues, and $I$ is the identity matrix. This system can be expressed as a polynomial

$$
\lambda^{2}-\left(v_{x x}+v_{y y}\right) \lambda+\left(v_{x x} v_{y y}-v_{x y} v_{y x}\right)=0 .
$$




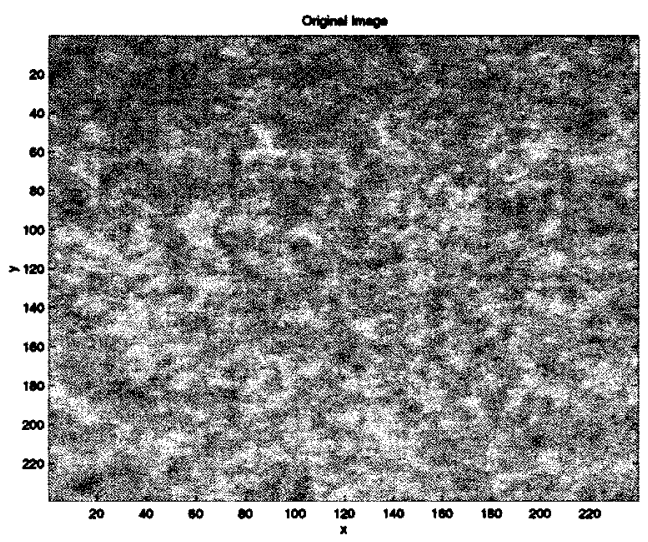

(a)

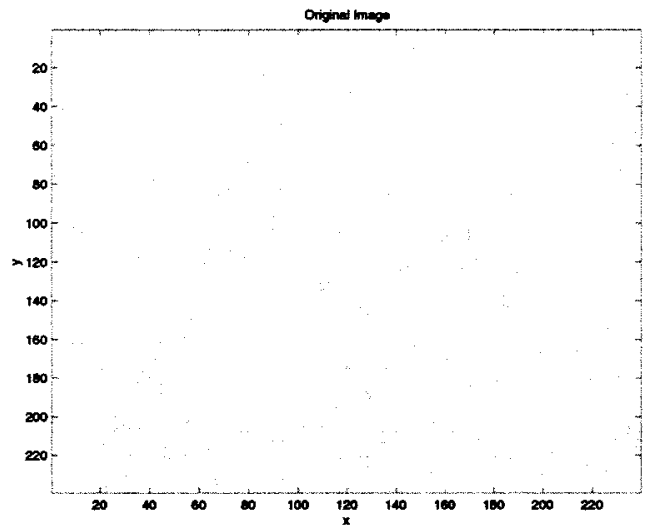

(c)

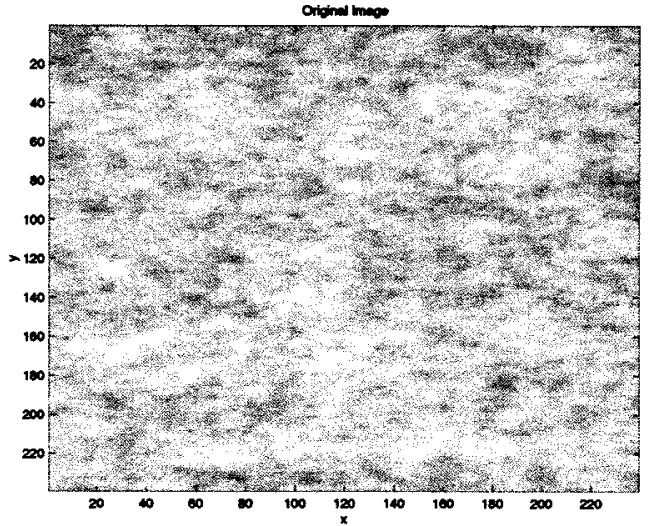

(b)

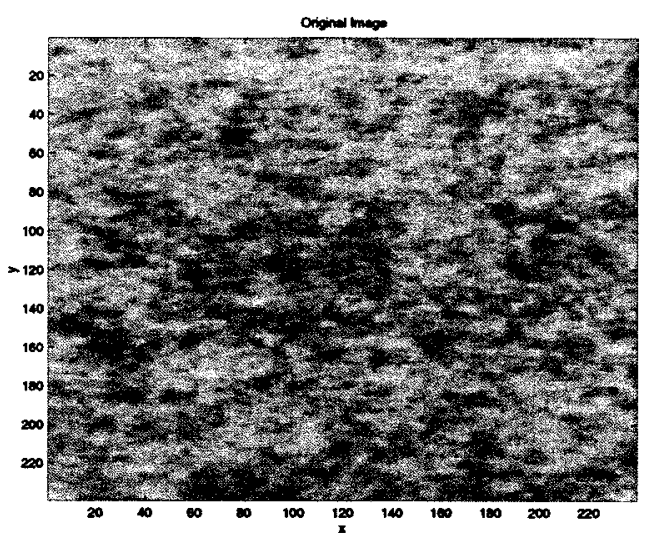

(d)

Fig. 2. Images of $\beta$-radiographs: (a) md1j44a1 and (b) pxxe50c1; and light transmission: (c) md1j44a1 and (d) pxxe50c1.

Finally, the eigenvalues are calculated as

$$
\begin{aligned}
& \lambda_{\max }=\frac{\left(v_{x x}+v_{y y}\right)+\sqrt{\left(v_{x x}-v_{y y}\right)^{2}+4 v_{x y}^{2}}}{2} \\
& \lambda_{\min }=\frac{\left(v_{x x}+v_{y y}\right)-\sqrt{\left(v_{x x}-v_{y y}\right)^{2}+4 v_{x y}^{2}}}{2}
\end{aligned}
$$

and the eccentricity $e$ is obtained using (7).

2) Spatial Variability of Local Anisotropy: In the previous section we discussed how anisotropy can be estimated as a global measure for the sample. However, sometimes it is also important to determine how anisotropy varies spatially within the sample.

Considering that eigenvectors describe axes of extremal variance of the $\overline{\phi(r, \Psi)}$ distribution of values, the eigenvalues $\lambda_{\min }$ and $\lambda_{\max }$ are related to the spatial variability of anisotropy along those axes. Indeed, strong anisotropy along a particular direction is characterized by a slow decay of $\overline{\phi(r, \Psi)}$ values, and a small eigenvalue associated with that direction.
We use as descriptors of anisotropy of spatial variability the reciprocal of the eigenvalues $\lambda_{\min }$ and $\lambda_{\max }$, here denoted simply as $\gamma_{\lambda_{\max }}$ and $\gamma_{\lambda_{\min }}$

$$
\begin{aligned}
& \gamma_{\lambda_{\max }}=\frac{1}{\lambda_{\max }} \\
& \gamma_{\lambda_{\min }}=\frac{1}{\lambda_{\min }}
\end{aligned}
$$

\section{Correlations and Spectra}

If we want to analyze the contents of images statistically, the entire image must be considered as a statistical quantity, namely, a random field. In this case, an $N \times M$ image consists of an $N \times M$ matrix whose elements are random variables. The gray values at two distinct positions can be related with each other by measuring their correlation. One measure for the correlation of the gray values is the expectation value for the product of the gray values at two positions, the autocorrelation function [9]

$$
\begin{aligned}
& R\left(x, y ; x+r_{x}, y+r_{y}\right) \\
& \quad=E\left\{I(x, y) I\left(x+r_{x}, y+r_{y}\right)\right\}
\end{aligned}
$$




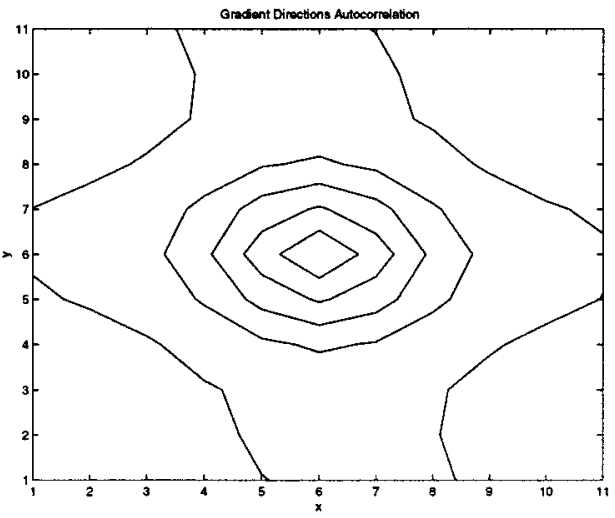

(a)

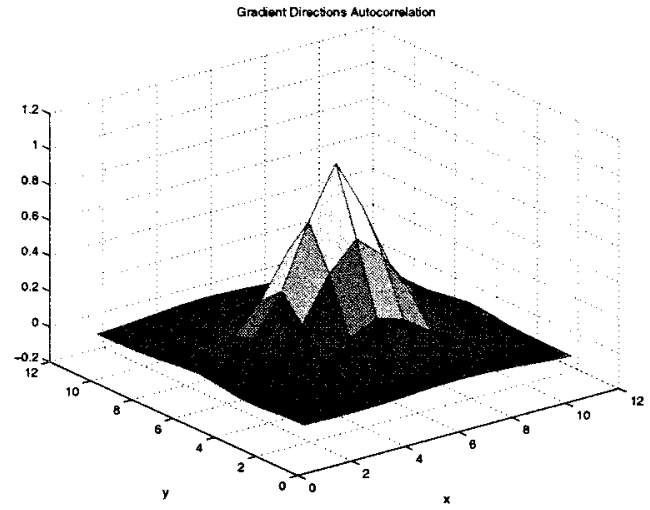

(c)

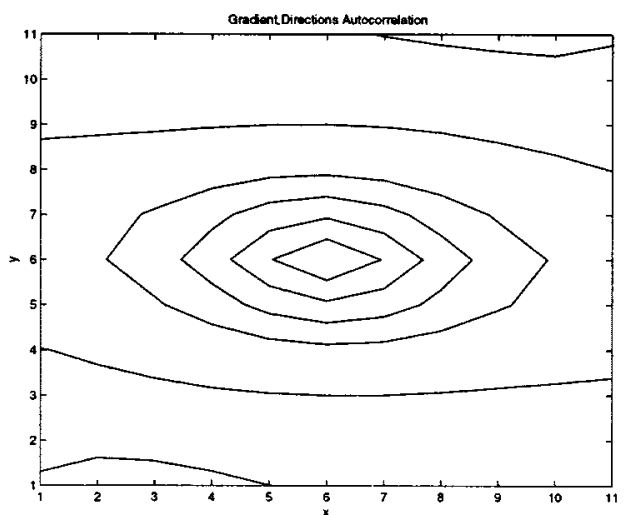

(b)

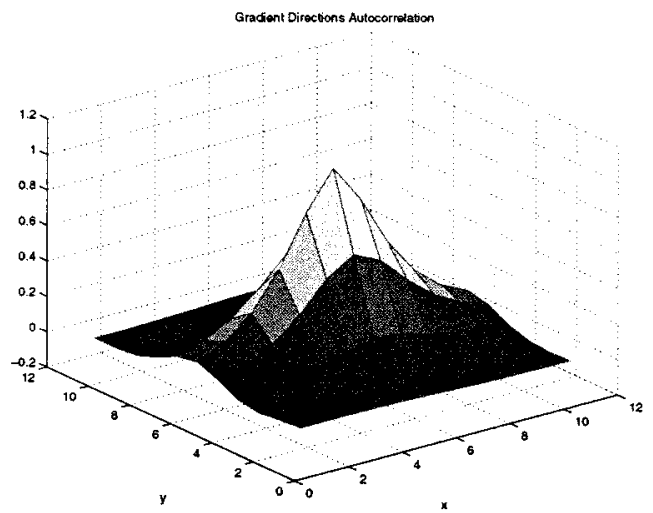

(d)

Fig. 3. Distribution of $\overline{\phi(r, \psi)}$ for $r \epsilon$ [1], [5], shown as surfaces: (a) md1j44a1 and (b) pxxe50c1; and shown as contours: (c) md1j44a1 and (d) pxxe50c1. $(\beta$-radiographs).

TABLE I

COMPARATIVE RESULTS FOR $e^{2}$ VALUES IN THE SAMPLES OF FIG. 2 FOR $\phi(r, \Psi)$ DATA

\begin{tabular}{c||c||c}
\hline Samples & $\beta$-radiographs & light transmission \\
\hline md1j44a1 & 2.5933 & 2.9130 \\
\hline pxxe50c1 & 17.4554 & 11.0301 \\
\hline
\end{tabular}

TABLE II

Comparative Results for $\gamma_{\lambda_{\max }}$ VALUES IN the SAMPLES OF FIG. 2 FOR $\phi(r, \Psi)$ DATA

\begin{tabular}{c||c||c}
\hline Samples & $\beta$-radiographs & light transmission \\
\hline md1j44a1 & 9.0089 & 9.0852 \\
\hline pxxe50c1 & 7.5396 & 7.8931 \\
\hline
\end{tabular}

TABLE III

Comparative Results FOR $\gamma_{\lambda_{\min }}$ VAlues IN THE SAMPLes of FIG. 2 FOR $\phi(r, \Psi)$ DATA

\begin{tabular}{c||c||c}
\hline Samples & $\beta$-radiographs & light transmission \\
\hline md1j44a1 & 11.4324 & 11.8691 \\
\hline pxxe50c1 & 15.4111 & 14.3844 \\
\hline
\end{tabular}

$$
=\sum_{m=0}^{Q-1} \sum_{n=1}^{Q-1} P\left(m, n ; x, y ; x+r_{x}, y+r_{y}\right)
$$

The probability function $P\left(m, n ; x, y ; x+r_{x}, y+r_{y}\right)$ has six parameters and tells us the probability of simultaneously measuring the gray value $m$ at the pixel $(x, y)$ and $n$ at the pixel $\left(x_{i}+m, y_{i}+n\right)$. If the statistics do not depend on the position of the pixel, the random field is called homogeneous. It implies that the mean value $\mu$ is constant over the whole image, and the autocorrelation function becomes shift-invariant. Therefore, the autocorrelation takes a simple form, and only depends on the positions of the pixels. Extending to two dimensions the Blackman-Tuckey method for autocorrelation estimation, we have

$$
\begin{aligned}
R\left(r_{x}, r_{y}\right) & =E\left\{I(x, y) I\left(x+r_{x}, y+r_{y}\right)\right\} \\
& =\frac{1}{N M} \sum_{x=0}^{2 N-2} \sum_{y=0}^{2 M-2} I(x, y) I\left(x+r_{x}, y+r_{y}\right)
\end{aligned}
$$

According to this method, the image $I(x, y)$ has size $N \times M$ and it is appended with zeros to the size $(2 N-1) \times(2 M-1)$ 


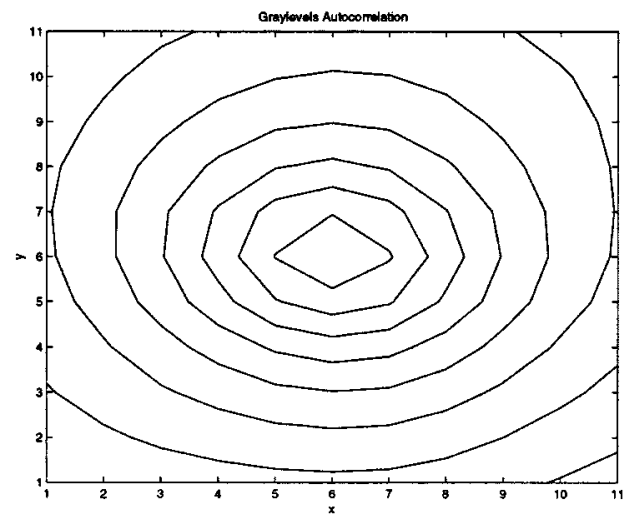

(a)

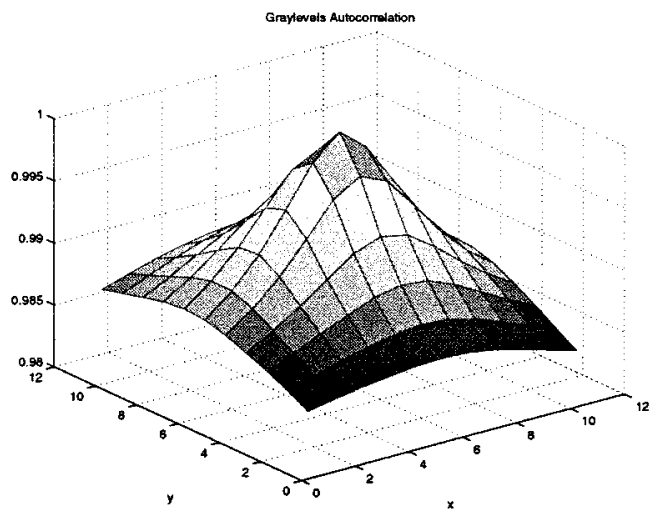

(c)

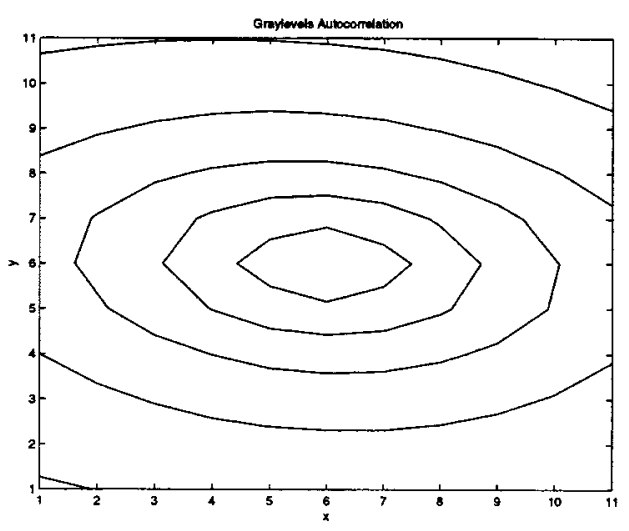

(b)

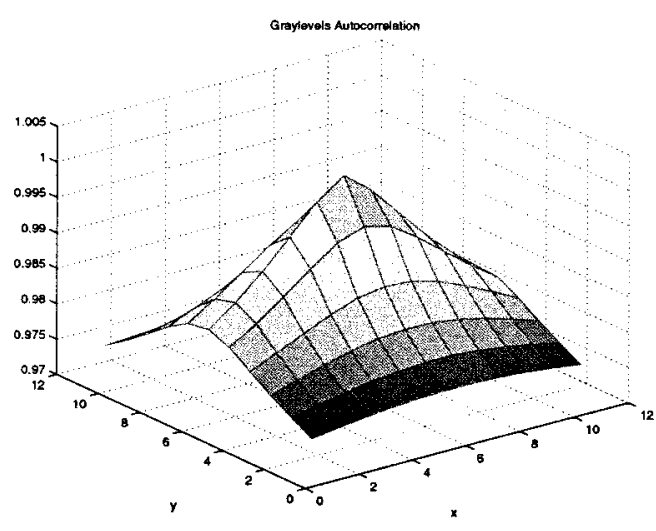

(d)

Fig. 4. Distribution of $R\left(r_{x}, r_{y}\right)$ for $r_{x}$ and $r_{y} \epsilon$ [1], [5], shown as surfaces: (a) md1j44a1 and (b) pxxe50c1; and shown as contours: (c) md1j44a1 and (d) pxxe50c1. ( $\beta$-radiographs).

before it is used in (16). If we subtract the mean $\mu$ from each pixel, we obtain the correlation

$$
\begin{aligned}
C\left(r_{x}, r_{y}\right)= & \frac{1}{N M} \sum_{y=0}^{M-1} \sum_{x=0}^{N-1} \\
& \cdot(I(x, y)-\mu)\left(I\left(x+r_{x}, y+r_{y}\right)-\mu\right)
\end{aligned}
$$

which is called autocovariance, and can be written in a simpler form as

$$
C\left(r_{x}, r_{y}\right)=R\left(r_{x}, r_{y}\right)-\mu^{2}
$$

In this case, the autocovariance is a linear function of the autocorrelation.

If the autocorrelation gradually decreases with the distance of the pixels, the pixels become more and more statistically independent. In this sense, the autocorrelation function is a description of the interrelation between the gray values of neighboring pixels. Therefore, if gray values of adjacent pixels have a stronger correlation along one direction, than along another direction, the random field is anisotropic. However, if correlation between gray values decreases equally in all directions, the random field is isotropic.
The Fourier transform of the autocorrelation function is the power spectrum $P\left(w_{x}, w_{y}\right)$, or spectral density, of the random field. It contains only information about amplitudes of the wave numbers of the Fourier transform, and can be associated to the energy in the wave number domain.

A stochastic texture image is often modeled as a sample of a first-order Markov process, where the correlation between points is proportional to their geometric separation. The autocovariance function for the two-dimensional (2-D) Markov process is [8]

$$
C\left(r_{x}, r_{y}\right)=K e^{-\sqrt{\alpha_{x}^{2} r_{x}^{2}+\alpha_{y}^{2} r_{y}^{2}}}
$$

where $K$ is an energy scaling constant and $\alpha_{x}$ and $\alpha_{y}$ are spatial decay constants.

The autocovariance function can be calculated for $r_{x}, r_{y}=$ $1,2,3, \cdots, L$, where $L$ is the correlation length considered. In this case, the parameters $\alpha_{x}, \alpha_{y}$ and $K$ have physical meaning.

The spatial decay constants $\alpha_{x}$ and $\alpha_{y}$ are related to the degree of anisotropy of the stochastic structure (i.e., if $\alpha_{x}=\alpha_{y}$ the structure is isotropic, and a deviation from this condition indicates an anisotropic structure). The energy scaling constant $K$ provides information regarding the degree of variability within 


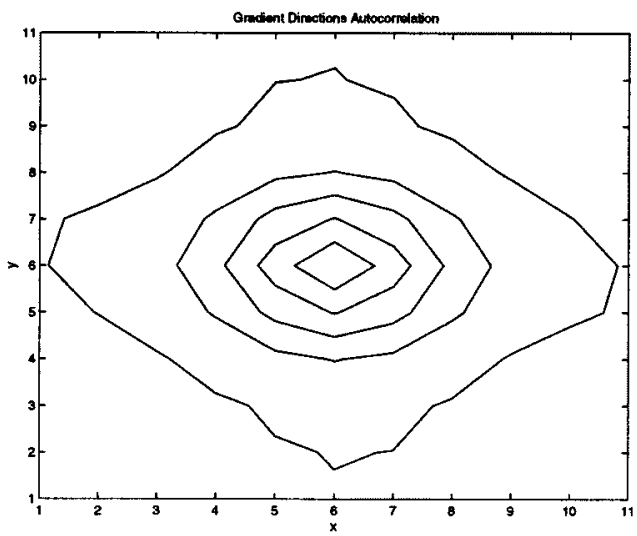

(a)

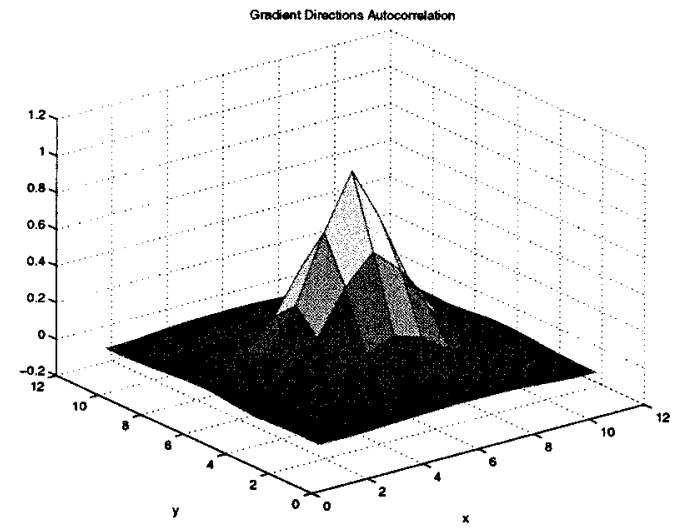

(c)

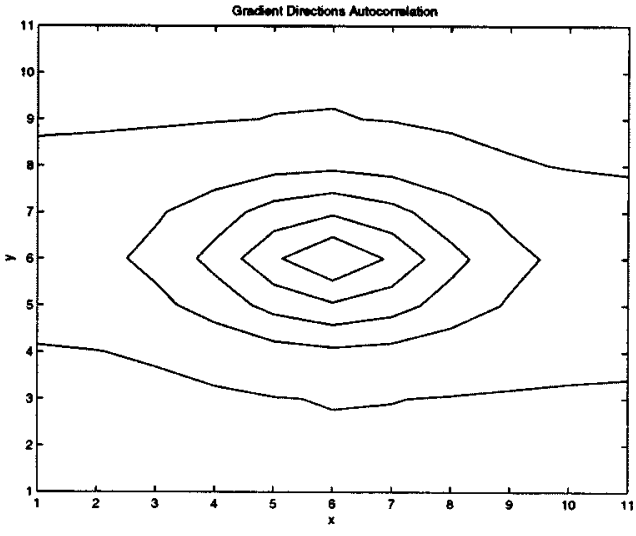

(b)

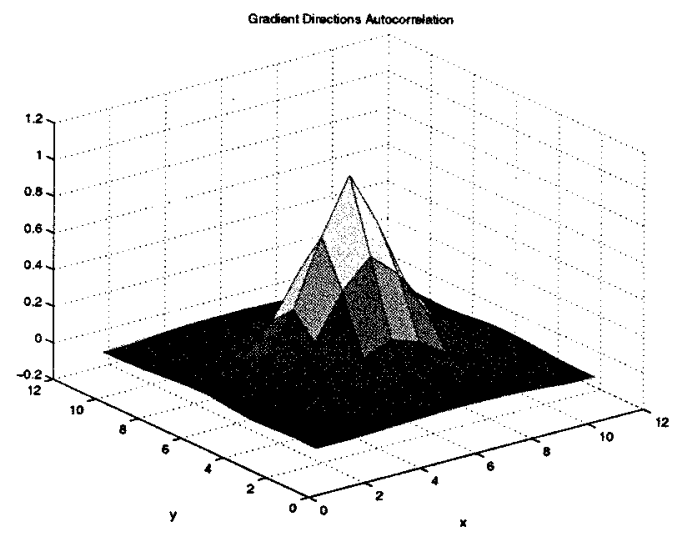

(d)

Fig. 5. Distribution of $\overline{\phi(r, \psi)}$ for $r \epsilon$ [1], [5], shown as surfaces: (a) md1j44a1 and (b) pxxe50c1; and shown as contours: (c) md1j44a1 and (d) pxxe50c1. (light transmission)

the structure (e.g., small $K$ reflects small gray values variance). These parameters can be obtained from the autocovariance function $C\left(r_{x}, r_{y}\right)$

$$
K=C(0,0)
$$

and

$$
\begin{aligned}
& \alpha_{x}^{2}=\frac{1}{r_{x}^{2}} \log ^{2}\left[\frac{C\left(r_{x}, 0\right)}{K}\right] \\
& \alpha_{y}^{2}=\frac{1}{r_{y}^{2}} \log ^{2}\left[\frac{C\left(0, r_{y}\right)}{K}\right] .
\end{aligned}
$$

The effect of noise can be reduced by taking the average of the estimates of $\alpha_{x}$ and $\alpha_{y}$, at various $r_{x}$ and $r_{y}$.

For the particular case of a real stochastic process, such as a stochastic texture image, and provided the autocovariance function, the power spectrum $P\left(w_{x}, w_{y}\right)$ can be estimated as follows:

$$
P\left(w_{x}, w_{y}\right)=\sum_{0}^{N-1} \sum_{0}^{M-1} C\left(r_{x}, r_{y}\right) \cos \left(r_{x} w_{x}+r_{y} w_{y}\right)
$$

and is expressed by

$$
P\left(w_{x}, w_{y}\right)=\frac{1}{\sqrt{\alpha_{x} \alpha_{y}}}\left[\frac{2 K}{1+\left[\frac{w_{x}^{2}}{\alpha_{x}^{2}}+\frac{w_{y}^{2}}{\alpha_{y}^{2}}\right]}\right] .
$$

The discussion above shows that there exists a relationship between the shape of the autocorrelation (and the autocovariance) function, and the corresponding power spectrum. Both functions have their decay controlled by the parameters $\alpha_{x}$ and $\alpha_{y}$. When $\alpha_{x}=\alpha_{y}$, the process represented is isotropic. However, if $\alpha_{x} \neq \alpha_{y}$ the process is anisotropic.

Therefore, methods proposed for anisotropy detection (e.g., the tensor method [9]) which are based on the shape analysis of the spectral density (such as fitting a straight line to detect orientation), actually measure anisotropy based on the shape of the autocorrelation function (or even on, the autocovariance function). Such methods are expected to suffer from limitations discussed in the next section. 


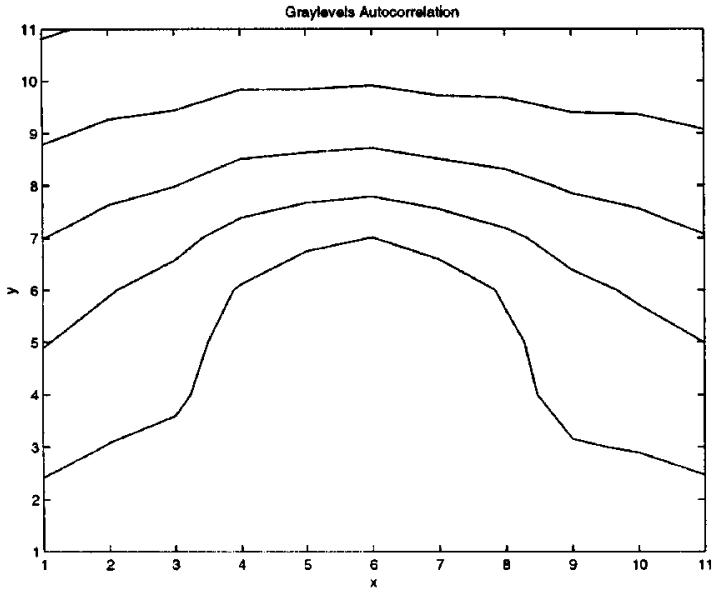

(a)

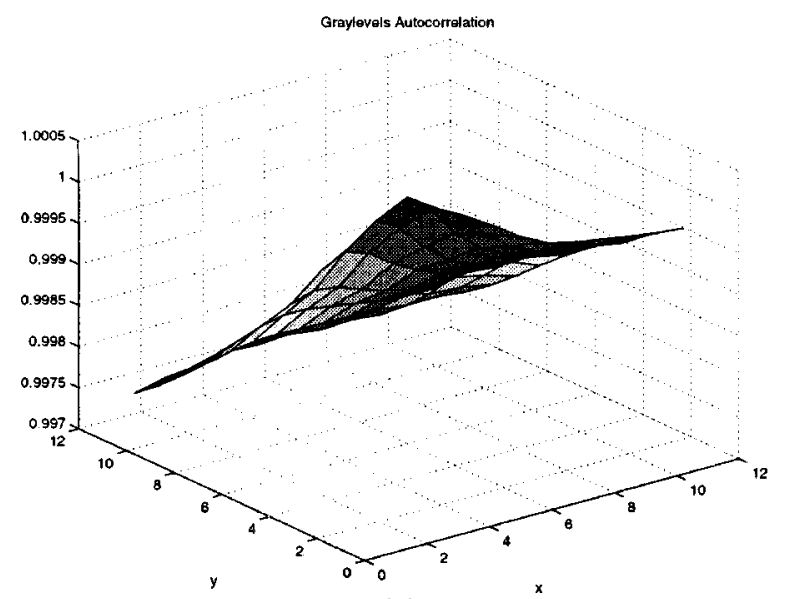

(c)

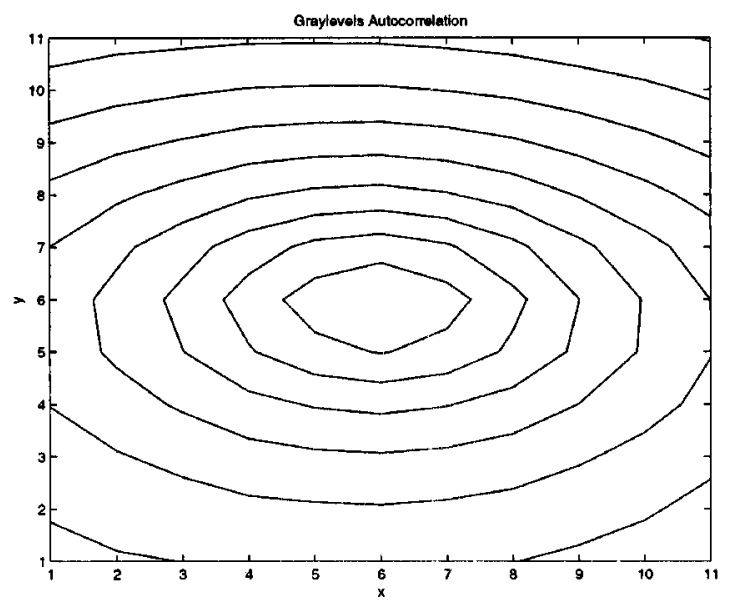

(b)

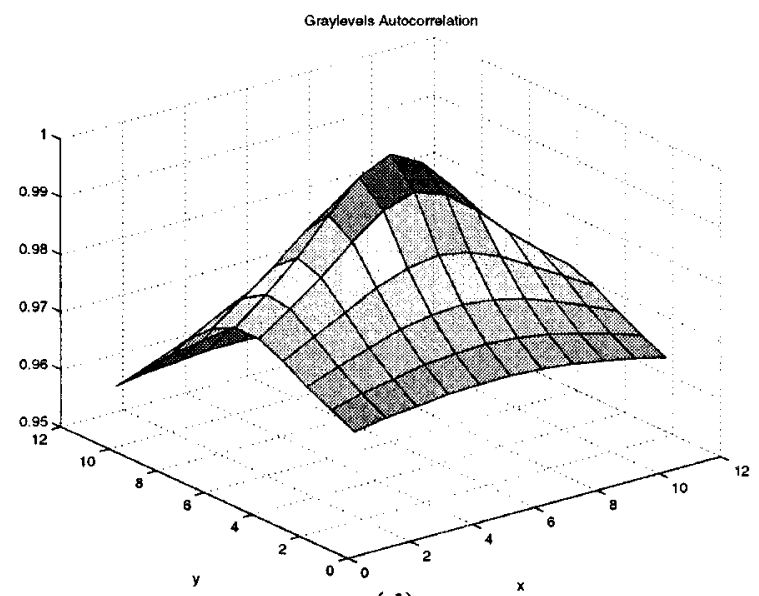

(d)

Fig. 6. Distribution of $R\left(r_{x}, r_{y}\right)$ for $r_{x}$ and $r_{y} \in$ [1], [5], shown as surfaces: (a) md1j44a1 and (b) pxxe50c1; and shown as contours: (c) md1j44a1 and (d) pxxe50c1. (light transmission)

TABLE IV

Comparative Results For $e^{2}$ VALUES IN THE SAMPLES OF Fig. 2 FOR $R\left(r_{x}, r_{y}\right)$ DATA

\begin{tabular}{c||c||c}
\hline Samples & $\beta$-radiographs & light transmission \\
\hline md1j44a1 & 1.0035 & 1.0003 \\
\hline pxxe50c1 & 1.0238 & 1.0296 \\
\hline
\end{tabular}

TABLE V

Comparative Results For $\gamma_{\lambda_{\max }}$ VAlues IN THE SAMPLes OF Fig. 2 FOR $R\left(r_{x}, r_{y}\right)$ DATA

\begin{tabular}{c||c||c}
\hline Samples & $\beta$-radiographs & light transmission \\
\hline md1j44a1 & 17.4953 & 17.3054 \\
\hline pxxe50c1 & 17.5887 & 17.6761 \\
\hline
\end{tabular}

TABLE VI

Comparative Results For $\gamma_{\lambda_{\min }}$ VALUES IN THE SAMPLES OF Fig. 2 FOR $R\left(r_{x}, r_{y}\right)$ DATA

\begin{tabular}{c||c||c}
\hline Samples & $\beta$-radiographs & light transmission \\
\hline md1j44a1 & 17.5255 & 17.3080 \\
\hline pxxe50c1 & 17.7968 & 17.9357 \\
\hline
\end{tabular}

\section{EXPERIMENTAL RESULTS AND DISCUSSION}

In order to illustrate the concepts developed, we analyzed the stochastic textures obtained through $\beta$-radiographic and optical density images (using light transmission), of paper samples for which data is in the public domain and accessible via a hypertext document on the World Wide Web pages: http://www.chem-eng.utoronto.ca/papersci/PaperSci.html.

These images have resolution of $1 / 4 \mathrm{~mm}^{2}$ per pixel, which was found experimentally to be sufficient to capture the texture structural information.

The stochastic texture samples utilized were supplied by J. Silvy, and have been studied in detail by different researchers [13]. The oriented sample selected, namely, pxxe50c1, was made on a pilot plant paper-machine of Fourdrinier type, at a medium to high level of flocculation. For comparison purposes, the sample md1j44a1 was also included, as an example of a well-formed nearly isotropic paper from a commercial paper-machine. The $\beta$-radiographic and light transmission images of these samples are in Fig. 2.

Similar trends are observed in the plots of the $\overline{\phi(r, \Psi)}$ distributions obtained from $\beta$-radiographs and light transmission im- 
ages, as shown in Figs. 3 and 5. It is visible in these figures that $\overline{\phi(r, \Psi)}$ decreases with $r$ more slowly along the directions $\Psi$ of higher anisotropy; on the other hand, $\overline{\phi(r, \Psi)}$ decreases almost uniformly with $r$ along all directions for the nearly isotropic sample. Also, the shape of these distributions presents nonuniformities [e.g., Figs. 3(b) and 5(b)] due to inhomogeneities in the paper structure.

The global anisotropy measured in the $\beta$-radiographic and light transmission images of these samples ( $e^{2}$ values) are shown in Table I. The $e^{2}$ descriptor appears to be consistent, and ranks the samples correctly in terms of their anisotropy. Also, the descriptors $\gamma_{\lambda_{\min }}$ and $\gamma_{\lambda_{\max }}$ may be found in Tables II and III. We clearly see the difference between the $\gamma_{\lambda_{\min }}$ and $\gamma_{\lambda_{\max }}$ values, measured for the anisotropic and the nearly isotropic samples. The $\gamma_{\lambda_{\min }}$ values are usually associated with the direction of stronger anisotropy, which corresponds to the $x$ coordinate in our examples.

The autocorrelation functions $R\left(r_{x}, r_{y}\right)$ of the texture images shown in Fig. 2 were calculated, and the performance of the proposed descriptors measured with these data. The shape of these functions are in Figs. 4 and 6, shown as contours and surfaces. It is evident that illumination changes, and/or structural defects, affect strongly the shape of those functions. For example, Fig. 6(a) and (c) reflects the shift in illumination (and/or light transmission) intensity along the field of view, in the $y$ direction. However, the shape of the $\overline{\phi(r, \Psi)}$ distribution was not significantly affected by this artifact, as Figs. 5(a) and (c) shows.

The decay of $R\left(r_{x}, r_{y}\right)$ values as a function of the distance $\left(r_{x}, r_{y}\right)$ is slower (between $3-5 \%$ ), than for $\overline{\phi(r, \Psi)}$ values (near 100\%). This indicates that larger areas are necessary to estimate $R\left(r_{x}, r_{y}\right)$, and also that descriptors based on these functions have a lower sensitivity to local variability. Tables IV-VI confirm that descriptor values are very similar for all textures, within the specified range of $\left(r_{x}, r_{y}\right)$ distances, and not allowing a clear discrimination between them with respect to their anisotropy (both samples appear to be nearly isotropic). Probably, this occurs because graylevels have a stronger spatial correlation than gradient orientations within a stochastic texture. For this reason, techniques based on the analysis of the shape of the autocorrelation function are expected to be less sensitive to local spatial variability, and also to be more influenced by illumination and/or structural artifacts than our approach.

The proposed descriptors contain information on anisotropy required for monitoring and control purposes. The analysis of the $\beta$-radiographic and light transmission images lead to similar results. This observation is of practical interest because it indicates that our technique has the potential to be applied either in laboratory, as well as, in on-line measurements.

Finally, we should mention that global anisotropy arises as locally oriented regions within the texture, as our results show.

\section{CONCLUding REMARKS}

A method for analyzing anisotropy in planar stochastic structures, as well as its spatial variability has been presented. This method has potential to be applied for monitoring and control of continuous web-making processes (e.g., papermaking machines), or for quality control purposes of 2-D monitoring. It is suitable for multiple resolution (i.e., different zone size) analysis of structural properties and the distribution of local anisotropy values.

\section{ACKNOWLEDGMENT}

The authors would like to thank Prof. J. Silvy for supplying some samples and $\mathrm{W}$. K. Ng for image acquisition and $\beta$-radiography.

\section{REFERENCES}

[1] J. Bigun, G. H. Granlund, and J. Wilklund, "Multidimensional orientation estimation with application to texture analysis and optical flow," IEEE Trans. Pattern Anal. Machine Intell., vol. 13, pp. 775-790, Aug. 1991.

[2] H. Corte, "The structure of paper," in Handbook of Paper Science, H. F. Rance, Ed. Amsterdam, The Netherlands: Elsevier, 1982, vol. 2, ch. 9.

[3] H. Corte and O. J. Kallmes, "Statistical geometry of fibrous networks," in Trans. 2nd Fundamental Research Symp. Oxford. London, U.K., Sept. 1961, pp. 13-46.

[4] J. Charrier and R. Marchessault, "Light scattering by random and oriented anisotropic rods," Fiber Sci. Technol., vol. 5, pp. 263-283, 1972.

[5] K. J. Niskanen and J. W. Sadowski, "Evaluation of some fiber orientation measurements," J. Pulp Paper Sci., vol. 15, no. 6, pp. 220-224, 1989.

[6] R. W. Perkins and R. E. Mark, "The role of fundamental research in papermaking," in Trans. 7th Fundamental Research Symp. Cambridge. Bury St Edmunds, Sept. 1981, 1983, pp. 479-526.

[7] Trans. 11th Fundamental Research Symp. Cambridge, C. F. Baker, Ed.. Leatherhead, Sept. 20-26, 1997, pp. 1293-1324.

[8] W. K. Pratt, Digital Image Processing. New York: Wiley, 1991.

[9] B. Jahne, Digital Image Processing: Concepts, Algorithms and Scientific Applications. Heidelberg, Germany: Springer-Verlag, 1995.

[10] C. Schaffnit, C. T. J. Dodson, and J. Silvy, "Orientation density distributions of fibers in paper," Nordic Pulp Paper Res. J., vol. 3, no. 7, pp. 121-125, 1992.

[11] C. Schaffnit and C. T. J. Dodson, "A new analysis of fiber orientation effects on paper formation," Paperija Puu., vol. 75, no. 9, pp. 68-75, 1993.

[12] J. Scharcanski and C. T. J. Dodson, "Texture analysis for estimating spatial variability and anisotropy in planar stochastic structures," Opt. Eng. J., vol. 35, no. 8, pp. 2302-2308, Aug. 1996.

[13] J. Silvy, "Etude Structurale de Milieux Fiberux: Cas des Fibers Cellulosiques," Doctorat D'Etat Es-Sciences Thesis, L'Institut National Polytechnique de Grenoble, 1980.

Jacob Scharcanski received the B.Eng. degree in electrical engineering and the M.Sc. degree in computer science from the Federal University of Rio Grande do Sul, Brazil, in 1981 and 1984, respectively, and the Ph.D. degree in systems design engineering from the University of Waterloo, Waterloo, ON, Canada, in 1993.

His main areas of interest are image processing and analysis, pattern recognition, and industrial automation. He was a Post-Doctoral Fellow at the Pulp and Paper Centre, University of Toronto, Toronto, ON, working on simulation of paper forming using neural networks, and on the analysis and interpretation of paper formation. Also, he was a Post-Doctoral Fellow at the Communications Group, Department of Electrical and Computer Engineering, University of Toronto, working on image processing and analysis. Currently, he is a Professor at the Institute of Informatics, Federal University of Rio Grande do Sul. He has authored and coauthored more than 60 publications in journals and conferences, and has led to innovations in paper structural analysis by combination of image analysis and statistical geometry. 
C. T. J. Dodson received the Ph.D. degree from Brunel University, U.K., in 1969.

He was a Visiting Professor at the vUniversity of Manchester, Institute of Science and Technology (UMIST), Manchester, U.K., University of Leuven, Belgium, University of Santiago-de-Compostela, Spain, and the University of British Colombia, Vancouver, BC, Canada, and currently is Professor of mathematics at UMIST. His research interests are in global differential geometry and topology, statistical geometry and their applications, and on formal mathematical theories alternative to topology for spaces of relations. These areas use computers for algebra in geometrical calculations and for graphics, and high-performance computers for simulation and characterization of stochastic structures. A large area of application of stochastic geometry and allied computations is in the study of multiphase flow and engineered stochastic structures like paper and nonwoven fabrics. He headed an NSERC/industrial research consortium, which is focused on the study of the structure and forming process of paper and has led to innovations in paper structural analysis by combination of image analysis and statistical geometry. He has authored or coauthored approximately 100 articles and 12 books on pure and applied geometry and calculus. 